\title{
Exercise and Its Effect on Bone Health
}

\author{
Paula J. Rackoff ${ }^{1}$, Jammie K. Barnes*,2 and Stephen Honig ${ }^{3}$ \\ ${ }^{I}$ Division of Rheumatology, Beth Israel Medical Center, 10 Union Sq East, Ste 3D, New York, NY 10003, USA \\ ${ }^{2}$ Internal Medicine Resident, Beth Israel Medical Center, 353 E $17^{\text {th }}$ St. Apt 18C, New York, NY 10003, USA \\ ${ }^{3}$ The Osteoporosis Center, The NYU Hospital for Joint Diseases-Orthopedic Institute, New York, NY. USA
}

\begin{abstract}
Peak bone mass is largely determined by genetic control, however, $30 \%$ is determined by environmental factors, most important exercise, nutrition and chronic illness. It is recognized that the maximizing peak bone density is essential in the reduction of fracture risk at all stages of life. For children and adolescents regular exercise particularly ones that include high impact features and a well balanced diet including milk based calcium and vitamin D is critical to maintaining and building strong bones. During the postmenopausal years, a regular schedule of physical activity emphasizing weight bearing exercises can help maintain bone mineral density and may reduce fracture risk.
\end{abstract}

\section{EXERCISE AND ITS EFFECT ON BONE HEALTH}

\section{Bone Growth and Development}

The mature adult skeleton contains about 1200 grams of calcium with the total bone mass increasing about 50 times between birth and maturity. Bone mass is largely driven by bone growth with men reaching a greater bone mass than women because their bones are, at skeletal maturity, larger not denser. The maximum amount of bone an individual accrues is called peak bone mass. While largely under genetic control, it is estimated that about $30 \%$ of peak bone mass is determined by environmental factors, most importantly nutrition, exercise and chronic illness. The age at which peak bone mass is achieved varies between areas of skeleton and among different populations. During adolescence and early childhood, bone mass increases until peak bone mass is fully attained during the third decade of life. There are gender differences in bone growth and development with females reaching peak bone mass earlier than males. Data from healthy girls indicate that bone mass gains are most pronounced between the ages 11 and 14 with the rates decreasing after the age 16 and/or two years after menarche [1]. In general, by the age of twenty, 90-96\% of peak bone mass has been attained. Exercise has been shown to help build bone mass in premenarchal girls during the main bone acquisition period of adolescence [2] and in older premenopausal women in their 40's and early 50 's. In a study involving young women 10-20 years of age that analyzed the contributions of exercise and calcium intake to bone mineral density values, Lloyd and colleagues found that achievable levels of exercise had a favorable effect on bone mineral density (BMD) and section modulus (bending strength) at the femoral neck and femoral shaft [3]. In contrast, calcium intake greater than $500 \mathrm{mg}$ daily had little effect on bone density values.

*Address correspondence to this author at the Internal Medicine Resident, Beth Israel Medical Center, 353 E $17^{\text {th }}$ St. Apt $18 C$, New York, NY 10003, USA; Tel: 212-420-2000; Fax: 212-844-8167; E-mail: jbarnes@chpnet.org

\section{Bone Cells and Muscle Function: The Example of Adolescence}

Considerable research has been done on the effect of exercise and hormonal status at the cellular level and at the functioning musculoskeletal level. Bone cells (osteoblasts, osteoclasts, and marrow stromal cells) are capable of responding to different types of mechanical signals. During remodeling, bone cells are stimulated by both gravity (osteoblast) and strain (osteocytes and stromal cells). The bone remodeling process is also influenced by the magnitude and the rate of strain as well as the load size and the number of loading cycles [4]. Recent evidence suggest that low intensity strains, when delivered at high frequency can be anabolic to bone; this finding has stimulated research defining how low magnitude, high frequency mechanical stimuli promotes bone growth, and how this data may relate to exercise induced bone growth [5]. In a study of young women with low bone mineral density Rubin and colleagues found that a one year program of low level whole body vibration $(30 \mathrm{~Hz}, 3 \mathrm{~g})$ resulted in an increase in cancellous $(2 \%)$ and an increase in cortical $(2.3 \%)$ bone compared to controls [6]. In addition, those women who were the most compliant users of the device had BMD increases of 3.9\% (cancellous) and 2.9\% (cortical) bone as well as a $7.2 \%$ increase in spinal musculature compared with controls and subjects who used the device irregularly. Rubin and colleagues concluded that extremely low levels of signals several orders less than generated by vigorous exercise increased bone and muscle mass in the weight bearing skeleton of young women with low BMD. The data in this study suggests that mechanical signals can influence both muscle and bone and that increased muscle contraction may produce anabolic strain on bone. In contrast, there is also data showing that too much strain on bone can induce micro damage and apoptosis adjacent to the damaged bone matrix [7].

The positive relationship between bone density and exercise in adolescent females has focused attention on the interactions between bone and muscle function. In adults, only small increases in bone mass have been achieved through a 
variety of exercise programs [8]. In children and adolescents, however, impressive gains in bone mass have been seen particularly in racket sports [9]. Muscle contraction results in bone deformation or strain and in developing muscles this strain may result in cortical bone growth presumably by stimulating osteoblast activity and growth factors [10]. The gains in bone mass seen during prepubescent female gymnast appear to be maintained for at least 20 years, despite a decrease in exercise intensity [11]. The mechanisms involved in exercise induced osteogenesis are not completely understood and may involve hormonal and/or mechanical influences. Physical activity during childhood and adolescence may account for up to $17 \%$ of the difference in bone mineral density among individuals [12]. Children participating in high intensity physical activity such as gymnastics and weight lifting show the greatest gains in bone mass. For this reason, the American College of Sports Medicine suggests the following programs for children and adolescents [13]:

Mode: Impact activities such as gymnastics, jumping (soccer, basketball)

Intensity: High in terms of bone loading with safety precautions

Frequency: At least 3 days per week

Duration: 10 to 20 minutes (two short sessions more effective)

\section{Estrogen, Nutrition and Vitamin D: Skeletal Impact}

Early and continuous exposure to endogenous estrogen during the bone growth potentiates peak bone mass in premenopausal women. In fact, studies have shown that early menarche is predictive of greater adult bone mass [14]. During adolescence, skeletal integrity of girls is largely dependent on hormone levels and impact exercise. Nichols and colleagues found significantly greater total hip and trochanteric BMD among eumenorrheic high school females engaged in high impact sports (soccer, softball, tennis, track sprinters or jumpers) compared to eumenorrheic female athletes participating in repetitive non-impact sports such as swimming, cross-country and track distance running [15]. In the same study, authors found that eumennorheic women engaged in high impact sports had greater spine and trochanteric BMD that did oligo/amennorheic athletes participating in the same sports.

Childhood and adolescence are stages of growth when significant musculoskeletal development occurs and it is consequently a time when adequate calcium and vitamin D are needed to fully maximize bone mass accrual. The most critical period for maximum bone mass formation is during puberty and early adolescence when there is $7-8 \%$ bone mass gain per year [16]. It is estimated that over a quarter of an adult's calcium is laid down during the two adolescent years of peak skeletal growth [17]. Female adolescents normally begin puberty between ages 9 and 10 and reach the period of maximum growth velocity at about age 12 . Unfortunately, the calcium intake among female teenagers begins to decline at the time of puberty and this decreased calcium intake often continues throughout the period of maximum growth [18]. The low calcium intake contrasts with the increased age-related need for this mineral and makes calcium deficiency a particular concern for female adolescents. There have been a number of population based studies that have analyzed calcium balance and calcium needs from infancy to young adulthood and these studies provide support for age related differences in the amounts of calcium needed for growth and development. The highest calcium requirements are during infancy and adolescence. The National Academy of Science recommends an average calcium intake of $1300 \mathrm{mg}$ for individuals ages nine to eighteen [19]. The relevance of this information was confirmed by Stear and colleagues who examined the relationship between adequate calcium intake and exercise on bone mineral content on a cohort of adolescent girls ages 16 to 18 and concluded that both calcium supplementation $(1000 \mathrm{mg} /$ day $)$ and exercise (45 minutes three times/week) significantly enhanced BMD when compared to a placebo control group [20]. Moreover, there is data that suggests that the need for calcium is even greater within two years of peak bone accrual during which time girls need $1500 \mathrm{mg}$ and boys $1700 \mathrm{mg}$ daily [21]. Estimating calcium intake is difficult and generally requires maintaining reliable food diaries over a finite period of time, something that is likely to be difficult for most adolescents in a community setting. Urinary calcium excretion increases with age during childhood and reaches its maximum by the end of puberty [22]. A study investigating the relationship between twenty-four hours urine calcium excretion and dietary calcium intake in adult women found this assay was not a reliable surrogate for estimating calcium intake [23]. As discussed maximizing peak bone mass accrual depends in part on adequate intake of calcium and vitamin D. Vitamin D insufficiency and deficiency are being increasingly recognized among all age groups. Inadequate dietary intake and widespread use of topical sunscreens are the underlying reasons for this phenomenon [24]. A recent three year prospective study involving healthy girls' ages 9 to 15 years showed that girls with hypovitaminosis D had significantly lower bone mineral density than the girls with normal levels of 25 hydroxyvitamin D [25]. The results suggest that pubertal girls with decreased levels of vitamin D are at risk for not reaching their maximum peak bone mass and that dietary enrichment or supplementation with vitamin D should strongly be considered for pubertal girls. While calcium supplements increase bone mass in children the effect does not persist once supplementation is discontinued. Kalkwarf and colleagues found that low milk intake during adolescence was associated with a $3 \%$ decreased in hip bone mineral content and BMD [26].

\section{Childhood Illness and Bone Health}

Chronic illnesses during childhood and adolescence can compromise bone health. Included among such illnesses are inflammatory bowel disease (IBD), the juvenile arthritides and childhood cancers. Decreased bone turnover has been documented in children with chronic illnesses. The cause of reduced bone mass in children with IBD has often been linked to the use of corticosteroids, but a study in 18 steroid naive children newly diagnosed with Crohn's disease found these children to have a significant incidence of low bone mass [27]. An earlier study by Boot and colleagues found that children with Crohn's disease had a higher risk of developing osteopenia that children with ulcerative colitis and that steroid therapy and nutritional status were also important determinants of BMD [28]. Sylvester and colleagues demonstrated in children with IBD that although 
indications of osteoblast activity increase with clinical improvement, bone mineral accrual does not necessarily accelerate in this population [29].

Murray et al. reported that $23 \%$ of individuals with systemic juvenile rheumatoid arthritis (JRA) had sustained at least one fracture; $56 \%$ of these were vertebral resulting in potentially permanent deformities [30]. The fractures were most common early in the disease process and $91 \%$ of the patients had received steroids. Periarticular osteoporosis is also a well recognized complication of the disease. Pepmueller et al. studied 41 children with JRA in a cross sectional study of and found clinical measures of disease activity correlated negatively with bone mass [31]. Henderson et al. found that measures of disease activity were significantly higher in prepubertal JRA children with low total BMD [32].

While major advances have been made in the treatment of childhood malignancies, comprise in BMD is a complication of both the disease and the treatments. Young adult survivors of childhood cancers are often exposed to prolonged bed rest, poor nutrition, gonadal failure and growth hormone deficiency. Exposure to drugs known to directly affect bone metabolism such as glucocorticoids, thyroid hormones, methotrexate and other potentially toxic chemotherapeutic agents is common. Aisenberg et al. showed that gonadal dysfunction was present in $40 \%$ of their patients and they documented a significant reduction in BMD when compared to healthy children of the same age [33]. Children with acute lymphoblastic leukemia and brain tumors have been clearly shown to be at risk because of bone marrow toxic drugs and whole body radiation [34]. Several studies have concluded that adult survivors of childhood cancers have reduced bone density and a trend towards routine screening of BMD in these patients at an earlier age is becoming well established.

\section{Exercise and Bone After the Adolescent Years}

Physical activity during adulthood helps maintain bone density. In postmenopausal women, regular exercise slows the rate of bone loss typical of this stage of life. It is difficult to determine precisely how much bone density is increased by physical activity; it is known, however, that these gains are lost when physical activity is discontinued. Kemmet and colleagues followed two groups of postmenopausal women for three years: one group participated in a vigorous training program; and the other group had no scheduled physical activity. Women who were active had no changes in bone density at the spine, hip and heel. However, women not in the physical activity group had significant decreases in bone density at all three sites [35]. Other studies have confirmed that physical activity produces minimal to modest increases in bone density; however, strength training and weight bearing exercises produces consistent and impressive decreases in falls and fractures. Qui et al. showed that Tai Chi improves neuromuscular function in the elderly and may have an effect on bone density [36]. In the Nurses Health Study, the group with the highest level of physical activity had $67 \%$ fewer hip fractures. Even women who did no other physical activity other than walking 4 hours per week reduced their risk of hip fracture by $41 \%$ [37].

\section{CONCLUSIONS}

It is increasingly recognized that maximizing peak bone density is essential in the reduction of fracture risk at all stages of life. While inherited factors account for most differences in bone mass, the modifiable determinants are also critically important. For children and adolescents regular exercise regimens, particularly those with high impact features and a well balanced diet containing adequate amounts of milk-based calcium, vitamin D and enough calories to support maximal skeletal growth and development is critical to building and maintaining strong bones. The relationships between exercise and bone mass accrual are complex; recent research shows promise of understanding how and when muscle activity can stimulate bone formation. For the present, it is clear that individuals across all ages: children, teenagers and adults should be encouraged to make regular exercise a part of their every day lives.

\section{REFERENCES}

[1] Soyka La, Fairfield WP, Klibanski A. Hormonal determinants and disorders of peak bone mass in children. J Clin Endocrin Metab 2000; 85: 3951-63.

[2] Petit MA, Hughes JM, Wetzsteon RJ, Novotny SA, Warren M. Weight-bearing exercise and bone mineral accrual in children and adolescents: a review of controlled trials. Bone 2007; 41(5): 9035.

[3] Lloyd T, Beck TJ, Lin HM, et al. Modifiable determinants of bone status in young women. Bone 2002; 30(2): 416-21.

[4] Diedenchs S, Freiberger F, van Griensven M. Effects of repetitive and short time strain in human bone marrow stromal cells. J Biomed Mater Res A 2008; [Epub ahead of print].

[5] Xie L, Jacobson JM, Choi ES. Low level mechanical vibrations can influence bone resorption and bone formation in the growing skeleton. Bone 2006; 39(5): 1059-66.

[6] Rubin C, Turner AS, Mallinckvodt C. Mechanical strain, induced noninvasively in the high frequency domain is anabolic to cancellous bone, but not cortical bone. Bone 2002; 30(3): 445-52

[7] Forwood MR. Mechanical effects on the skeleton: are there clinical implications? Osteoporosis Int 2001; 12(1): 77-83.

[8] Kemmler W, Engelke K, von Stengel S, et al. Long-term four-year exercise has a positive effect on menopausal risk factors: the Erlangen Fitness Osteoporosis Prevention Study. J Strength Cond Res 2007; 21(1): 232-9.

[9] Bass L, Saxon L, Daly RM, et al. The effect of mechanical loading on the size and shape of bone in pre-, peri-, and postpubertal girls: a study in tennis players. J Bone Mineral Res 2002; 17: 2274-80.

[10] Scott A, Khan KM, Duronio V, et al. Mechanotransduction in human bone: in vitro cellular physiology that underpins bone changes with exercise. Sport Med 2008; 38(2): 139-60.

[11] Zanker CL, Osborne CB, Cooke B, et al. Bone density, body composition and menstrual history of sedentary female former gymnast, aged 20-32 years. Osteoprosis Int 2004; 15(2): 145-54.

[12] Welten DC, Kemper HC, Post GB, et al. Weight-bearing activity during youth is a more important factor for peak bone mass than calcium intake. J Bone Mineral Res 1994; 9(7): 1089-96.

[13] Kohrt WM, Bloomfield SA, Little KD, et al. American College of Sports Medicine Position Stand: physical activity and bone health. Med Sci Sports Exerc 2004; 36(11): 1985-96.

[14] Chevalley T, Rizzoli R, Hans D, et al. Interaction between calcium intake and menarcheal age on bone mass gain: 8-year follow-up study from pre-puberty to post-menarche. J Clin Endocrinol Metab 2005; 90(1): 44-51.

[15] Nichols JF, Sauh MJ, Barrack MT, Barkal H-S. Bone mineral density in female high school athletes: interactions of menstrual function and type of mechanical loading. Bone 2007; 41: 371-7.

[16] Lloyd T, Beck TJ, Lin HM, et al. Modifiable determinants of bone status in young women. Bone 2002; 30(2): 416-21.

[17] Whiting SJ, Vatanparast H, Baxter-Jones A, et al. Factors that affect bone mineral accrual in the adolescent growth spurt. J Nutr 2004; 134(3): 6965-7005.

[18] Lloyd T, Beck TJ, Lin HM, et al. Modifiable determinants of bone status in young women. Bone 2002; 30(2): 416-21.

[19] Bryant RJ, Cadogan J, Weaver CM. The new dietary reference intakes for calcium: implications for osteoporosis. J Am Coll Nutr 1999; 18(5 Suppl): 406S-12S. 
[20] Stear SJ, Prentice A, Jones SC, et al. Effect of a calcium and exercise intervention on the bone mineral status of 16-18-y-old adolescent girls. Am J Clin Nutr 2003; 77(4): 985-92.

[21] Molgaard C, Thomsen BL, Michaelsen KF. Whole body bone mineral accretion in healthy children and adolescents. Arch Dis Child 1999; 81: 10-15.

[22] Matkovic V. Calcium Metabolism and calcium requirements during skeletal modeling and consolidation of bone mass. Am J Clin Nutr 1999; 54(1Suppl): 245S-60S.

[23] Toren PJ, Norman RW. Is 24-hour urinary calcium a surrogate marker for dietary calcium intake? Urology 2005; 63: 459-62.

[24] Sinclair C. Risks and benefits of sun exposure; implications for public health practice based on Australian experience. Prog Biophys Mol Biol 2006; 92(1): 173-8.

[25] Lehtonen-Veromaa MK, Mottonen TT, Nuotio, et al. Vitamin D and attainment of peak bone mass among peripubertal Finnish girls: a 3-y prospective study. Am J Clin Nutr 2002; 76(6): 144653.

[26] Kalkwarf HJ, Khoury JC, Lamphear BP. Milk intake during childhood and adolescence, adult bone density, and osteoporotic fractures in US women. Am J Clin Nutr 2003; 77(1): 257-65.

[27] Harpavat M, Greenspan SL, Obnene C, et al. Altered bone mass in children at diagnosis of Crohn's disease: a pilot study. J Pediatr Gastroenterol Nutr 2005; 40: 295-300.

[28] Boot AM, Bouquet J, Krenning EP de Muinck-Schrama SM. Bone mineral density and nutritional status in children with chronic inflammatory bowel disease. Gut 1998; 42: 188-94.

[29] Sylvester FA, Wyzga N, Hyams JS, et al. Natural history of bone metabolism and bone mineral density in children with inflammatory bowel disease. Inflamm Bowel Dis 2007; 13(1): 42-50.
[30] Murray KJ, Boyle RJ, Woo P. Pathological fractures and osteoporosis in a cohort of 103 systemic onset juvenile idiopathic arthritis patients. Arthritis Rheum 2000; 43: S119

[31] Pepmuellen PH, Cassidy JT, Allen SH, et al. Bone mineralization and bone mineral metabolism in children with JRA. Arthritis Rheum 1996; 39: 746-57.

[32] Henderson CJ, Cawkwell GD, Specker BL, et al. Predictors of total body bone mineral density in non-corticosteriod treated prepuberty children with juvenile rheumatoid arthritis. Arthritis Rheum 1997; 40: 1967-75.

[33] Aisenberg J, Hsieh K, Kalaitzoglou G, et al. Bone mineral density in young adult survivors of childhood cancer. J Pediatr Hematol Oncol 1998; 20: 241-5.

[34] Arikoski P, Komalainen J, Voutilainen R, et al. Reduced bone mineral density in long term survivors of childhood acute lymphoblastic leukemia. J Pediatr Hematol Oncol 1998; 20: 234-40.

[35] Kemmler W, Engelke K, von Stengel S, et al. Long-term four-year exercise has a positive effect on menopausal risk factors: the Erlangen Fitness Osteoporosis Prevention Study. J Strength Cond Res 2007; 21(1): 232-9.

[36] Qin L, Choy W, Leung K. Beneficial effects of regular Tai Chi exercise on musculoskeletal system. J Bone Miner Metab 2005; 23(2): 186-90.

[37] Feskanich D, Willett W, Colditz G. Walking and leisure-time activity and risk of hip fracture in postmenopausal women. JAMA 2002; 288(18): 2300-6.

(C) Rackoff et al.; Licensee Bentham Open.

This is an open access article licensed under the terms of the Creative Commons Attribution Non-Commercial License (http://creativecommons.org/licenses/by-nc/3.0/) which permits unrestricted, non-commercial use, distribution and reproduction in any medium, provided the work is properly cited. 